\title{
A non-pharmacological intervention to manage behavioral and psychological symptoms of dementia and reduce caregiver distress: Design and methods of project $\mathrm{ACT}^{3}$
}

\author{
Laura N Gitlin' \\ Laraine Winter' \\ Marie P Dennis' \\ Walter W Hauck ${ }^{2}$ \\ 'Center for Applied Research on \\ Aging and Health (CARAH), Thomas \\ Jefferson University, Philadelphia, \\ PA, USA; ${ }^{2}$ Formely Division of \\ Biostatistics, Department of \\ Pharmacology and Experimental \\ Therapeutics, Thomas Jefferson \\ University, Philadelphia, \\ PA, USA. Currently Sycamore \\ Consulting, LLC New Hope, PA, USA; \\ ${ }^{3}$ Funded by the National Institute \\ on Aging and the National Institute \\ on Nursing Research (Grant \# ROI \\ AG22254). Clinical trial registration \\ \#NCT00259480.
}

\begin{abstract}
Project ACT is a randomized controlled trial designed to test the effectiveness of a non-pharmacological home-based intervention to reduce behavioral and psychological symptoms of dementia (BPSD) and caregiver distress. The study targets 272 stressed racially diverse family caregivers providing in-home care to persons with moderate stage dementia with one or more behavioral disturbances. All participants are interviewed at baseline, 4-months (main trial endpoint), and 6-months (maintenance). The four-month intervention involves up to 13 visits from an occupational therapist who works with families to problem-solve potential triggers (communication style, environmental clutter) contributing to behaviors, and instruct in strategies to reduce caregiver stress and manage targeted behaviors. To rule out infection or other potential medical contributors to behaviors, a nurse obtains blood and urine samples from the dementia patient, and conducts a medication review. Participants in the no-treatment control group are offered the nurse arm and one in-home session following trial completion at 6-months. This paper describes the research methods, theoretical and clinical aspects of this multi-component, targeted psycho-social treatment approach, and the measures used to evaluate quality of life improvements for persons with dementia and their families.
\end{abstract}

Keywords: family caregiving, environmental modification, home care, occupational therapy, psychosocial intervention

Over 4 million people in the United States have Alzheimer's disease or a related disorder, the vast majority of who are cared for at home by family members (Haley and Bailey 1999). Integral to dementia is a broad spectrum of behavioral manifestations including agitative and disruptive behaviors and depressive affect (Lyketsos, Breitner et al 2001; Lyketsos, Sheppard et al 2001). Referred to as behavioral and psychological symptoms of dementia (BPSD), the etiology of behaviors and the relationship to pathology remain unclear (Swearer et al 1988; Schneider et al 1990; Colenda 1995; Swanwick 1995; Finkel and Burns 2000). Although some studies show that the prevalence and severity of behaviors increase with global severity of dementia; others suggest a non-monotone pattern with the most disturbing behaviors (aggressiveness) occurring at the moderate stage of the disease and diminishing with disease progression (McCarty et al 2000).

Prevalence rates of BPSD among community-living patients vary considerably across studies from $58 \%$ to $90 \%$, although most report that close to $80 \%$ of patients manifest at least one disruptive behavior over the course of the disease (Reisenberg 
et al 1987; Cohen-Mansfield et al 1989). A study of 126 ambulatory, community living patients found that $83 \%$ exhibited at least one of the following behaviors: $51 \%$ had angry outbursts, $45 \%$ had disturbed sleep, and 21\% had assaultive or violent episodes (Swearer et al 1988). Another study of 96 community-residing dementia found that $72 \%$ of dementia patients asked the same questions repeatedly, 34\% woke up at night, 25\% were verbally abusive and $20 \%$ refused care (Baumgarten et al 1990).

Behavioral occurrences not only compromise the wellbeing of persons with dementia, but have a profound effect on family caregivers (Ballard et al 2000). Research consistently shows that behaviors such as resisting care or wandering are the most distressing and financially costly aspect of the disease to caregivers, often contributing to the decision to place a family member in a nursing home. Interventions to help families effectively manage challenging behaviors at home have not been systematically evaluated and there is no clinical pathway that is typically followed by health professionals to address this aspect of the disease. Early efforts to manage behaviors focused chiefly on pharmacological approaches, with only small benefits demonstrated for specific types of behaviors (Burton et al 1995; Schneider et al 1990). Although a pharmacological approach can be effective in managing certain psychiatric behaviors (eg, hallucinations, paranoia, depression), for some patients, overt behavioral disturbances such as wandering, resistance to care, agitation, or repetitive vocalizations, are less amenable to drug management (Class et al 1997).

Current research suggests that behavioral disturbances can not be explained solely by diminished cognitive capacity but rather, reflect the interplay or interaction between the pathology, a person's capabilities and the physical and social environment in which the behavior occurs (Boucher 1999; Mittelman 2000; Cohen-Mansfield 2001; MonizCook and Vernooij-Dassen 2006). The premise is that persons with dementia become increasingly vulnerable to their environment and have progressively lower thresholds to processing external stimuli. This discrepancy between capabilities and environmental demands heightens negative behavioral outcomes. As such, one approach to managing behaviors is modifying environmental factors that place too much demand or press on the individual. Environmental modification has been shown to be effective in addressing agitation in nursing home settings (Gitlin et al 2003; Calkins 1989), but with few exceptions, has not been systematically tested to address occurrences of BPSD in the home (Gitlin et al 2001, 2003).
Project ACT (Advancing Caregiver Training) is a randomized two-group controlled trial to test the efficacy of a multi-component home-based intervention that modifies the environment and other potential behavioral triggers to reduce the frequency of occurrence of targeted behaviors and associated caregiver distress.

\section{Primary research aims}

The primary aims of this study are to test the: (1) immediate (4-months) effectiveness of the intervention to reduce the frequency of occurrence of targeted disruptive behaviors in persons with dementia and to reduce caregiver upset; and (2) maintenance of treatment effects at 6-months. We also propose several exploratory aims. If the intervention has a positive effect, we will evaluate the mechanisms of action, or pathways by which treatment gains are obtained. Specifically, we plan to evaluate whether caregiver skill acquisition (eg, use of positive communication and task simplification techniques) mediates treatment outcomes. Second, given that previous research shows that caregiver characteristics may moderate treatment outcomes, we plan to evaluate whether there is a differential treatment effect based on characteristics of the patient and caregiver (eg, gender, race, relationship, and cognitive status) (Baron and Kenny 1986). Finally, an additional study arm has been added that enrolls control group participants to receive the nurse intervention only. This will allow us to evaluate whether identifying and minimizing medical contributors to behaviors has an effect on behavioral occurrence reduction independent of the caregiver skill enhancement and environmental redesign components of the intervention.

\section{Overview of study design}

Using a two-group randomized, parallel design, 272 caregivers will be enrolled and assigned to either treatment or a usual care control group (Figure 1). Families are being recruited through area social agencies in the Philadelphia region and media announcements. Interested caregivers contact the research team by telephone or mail-in a self-addressed and stamped post-card attached to the study brochure. Interested persons are screened for eligibility and willingness to participate using a brief telephone screen. Family caregivers who are eligible and willing to participate are interviewed at home by a trained interviewer and sign an IRB approved informed consent form. Following a baseline (T1) assessment, caregivers are randomized using a stratified, permuted block design based on caregiver relationship to the individual with dementia (spouse/non-spouse). We seek 


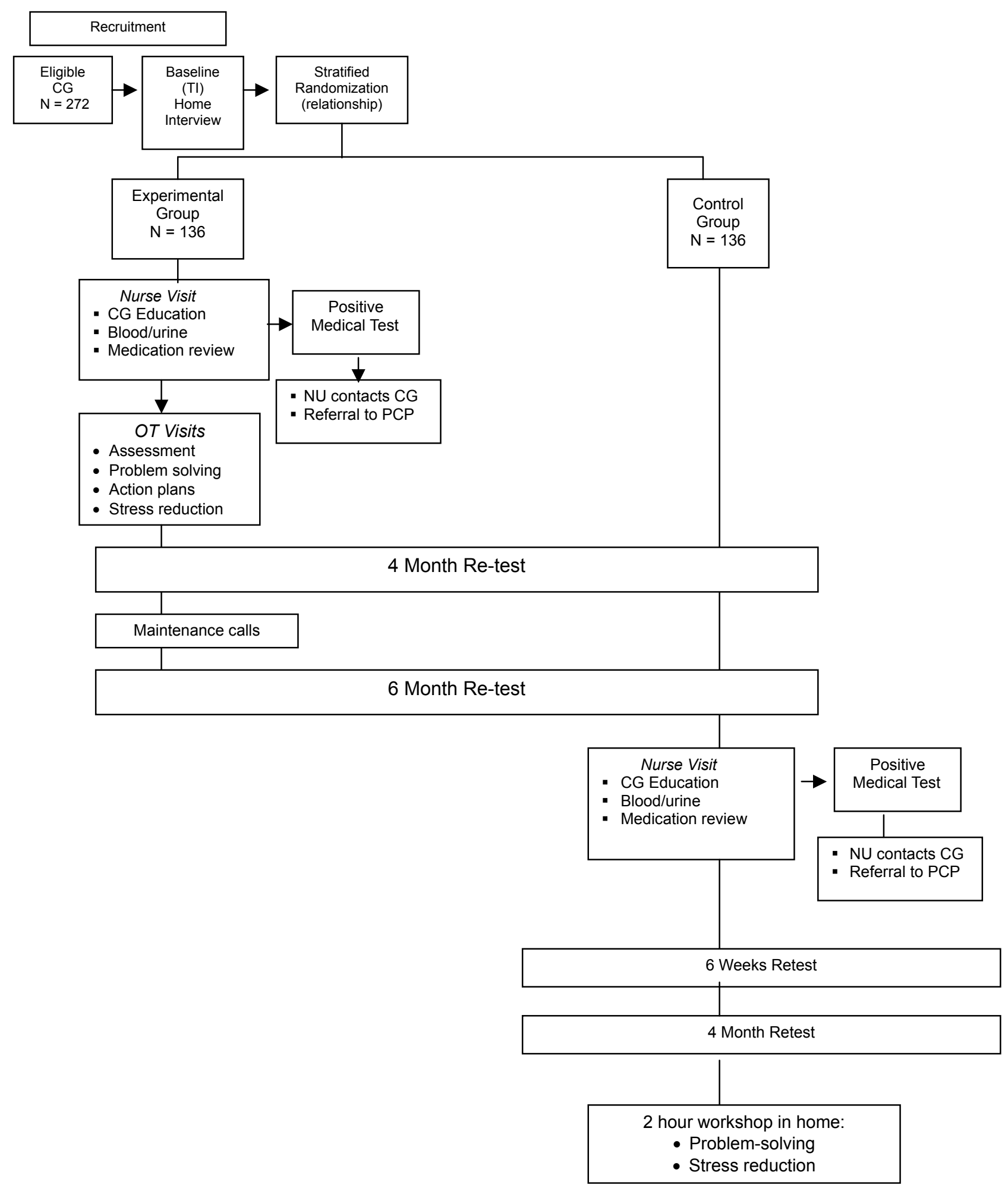

Figure I Project ACT study design with additional arm.

Abbreviations: CG, caregiver; OT, occupational therapist; NU, nurse; PCP, primary care physician.

to assure assignment balance in view of previous research showing differential treatment outcomes by relationship to patient. Intervention caregivers receive up to 13 contacts with nurse and occupational therapist interventionists. All study participants are reassessed at 4 and 6-months from baseline by trained interviewers who remain masked to study assignment. Usual-care control-group participants do not receive intervention contact but can elect to participate in 
the nursing arm of the intervention at the conclusion of their 6-month participation in the main randomized trial. This is an additional arm of the randomized trial that was developed after the study was initiated. We added this arm based on preliminary data showing higher rates of undetected medical problems than originally expected among intervention participants based on the nurse assessment. Control participants who elect to participate in the nurse arm of the intervention are reevaluated at 6-weeks and then 4-months from T3 to determine the effect of this one intervention component on behavior reduction. Following the T3 reassessment, control participants can elect to participate in a 2 hour home educational workshop to learn problem solving and stress reduction techniques. Control participants who choose not to participate in the additional treatment arm are offered the 2 hour home workshop following completion of their 6-month retest.

Criteria for study eligibility involve both the caregiver and person with dementia. Caregivers are eligible for study participation if they are: (1) caring at home for a person who either has a physician diagnosis of dementia or a Mini-mental State Examination (MMSE) score of less than 23 (Folstein and Bylsma 1999); (2) a family member 21-years of age or older (male or female); (3) English speaking; (4) planning to live in the area for 6-months and not actively seeking nursing home placement; and (5) reporting the occurrence of one or more behaviors using the 16-item Agitated Behavior in Dementia Scale (ABID) (Logsdon et al 1999) and high level of personal upset. Caregivers are excluded if the caregiver or dementia patient: (1) has a terminal illness with life expectancy less than 6-months; (2) is in active treatment for cancer; or (3) had greater than 3 acute medical hospitalizations in the past year; or (4) is currently involved in another clinical trial of psychosocial or educational support. Also, caregivers are excluded if their family member: (1) has schizophrenia or a bi-polar disorder; (2) has dementia secondary to probable head trauma; (3) has an MMSE score of 0 and are bed-bound (eg, confined to bed or chair for at least 22 hours a day for at least four of the previous seven days), and thus excludes caregivers of patients at the most severe stage of the disease who may not benefit from the intervention; and (4) is enrolled in a clinical trial involving pharmacological treatment for agitation or other behaviors.

\section{Project ACT intervention}

Four principles guided the development of the Project ACT intervention: (1) the intervention had to be theorybased; (2) its components had to be empirically derived and/or reflect best-documented clinical practice; (3) the intervention components had to be either reimbursed under current Medicare and insurance practices or fundable through existing government caregiver support programs to enhance its transportability into real-world care of dementia patients and their caregivers; and (4) the intervention had to be portable and replicable such that all protocols are standardized and materials for the intervention can easily be replicated and disseminated. The condition of reproducibility is essential if the intervention is to be clinically useful and made available in primary care, home care or long-term health care practices.

\section{Conceptual frameworks}

The Project Act intervention draws upon several theoretical frameworks. First, we use a stress health process framework to explain the stressful impact of BPSD on family members (Ballard et al 2000; Schulz et al 2000). According to this model, as applied to behavioral disturbances, primary stressors include patient behaviors and social and environmental stressors (eg, multiple role demands, over or under stimulating environment). The model suggests that caregivers evaluate whether these demands pose a threat and, if so, whether they have sufficient coping mechanisms to minimize the threat. If caregivers perceive demands as threatening and coping resources as inadequate, they may become burdened and at increased risk of physical and psychiatric illness. The goal of Project ACT is to enhance the coping resources of caregivers through skills training and minimize external stressors (medical and environmental) contributing to the appraisal of upset. If caregivers have coping resources and the skills to effectively manage behaviors, they may experience enhanced mastery and less generalized burden.

To enhance the coping resources of caregivers to identify and modify potential triggers of behaviors, we draw upon environmental models such as Lawton and Nahemow's competence-environmental press framework (Lawton and Nahemow 1973). This framework suggests that the interaction between an individual's competence and press (environmental supports and restrictions) of the environment shapes behavior (Lawton 1982). Hall and Buckwalter (1987) further suggest that persons with dementia experience a progressively lowered stress threshold due to their declining abilities to cope with external stimuli resulting in increasing anxiety and inappropriate behaviors. Therefore, minimizing environmental stressors may prevent the accumulative build up of negative effects from a demanding environment; this in turn, may prevent, reduce or minimize disruptive behaviors. In previous research, we have developed and tested specific 
strategies that reflect low cost manipulations to the home environment (Corcoran and Gitlin 1991). For example, removal of auditory clutter and excess objects in a room can decrease confusion and disorientation that may contribute to agitation. Grab bars and tub seats may simplify bathing routines and decrease fear thus minimizing resistance to care. Enhancing caregiver communication skills and ability to set up daily predictable and simplified routines may prevent catastrophic reactions.

\section{Intervention process}

The intervention involves an active and maintenance phase over a 6-month period as shown in Table 1 . The active phase occurs over 4-months involving up to 11 contacts $(90$ minute in-home visits and 20 minute telephone contacts) spaced such that caregivers have opportunities to practice strategies that are introduced first with a health professional and then independently. The occupational therapist (OT) initiates the intervention by introducing the goals of the intervention and conducting an assessment of the home environment for safety, support of daily function and ease of navigation, caregiver concerns and management style, and caregiver-patient interactions. Following this initial visit, an advanced practice nurse (NU) meets with the family caregiver and provides and reviews educational materials on dementia, the importance of taking care of oneself as a caregiver (eg, NIA 2002 booklet, Caregiver Guide: Tips for Caregivers of People with Alzheimer's Disease), and medical conditions that may contribute to or exacerbate behaviors. The NU also obtains blood and urine samples from the dementia patient and reviews their medications. For the urine test, a dip stick is used to determine glucose (diabetes out of control), blood in urine (possible urinary track infection), other possible diseases, and elevated $\mathrm{pH}$ or the presence of nitrates (indicative of urinary tract infection). Dehydration can also be indicated by high specific gravity, dark color of urine, or strong odor; uncontrolled diabetes, a urinary tract infection or dehydration. If blood or nitrates are found in the urine or $\mathrm{pH}$ is elevated, the specimen is sent to a laboratory for culture and sensitivity. From the blood sample, a complete blood count (CBC) is obtained to rule out anemia, infection. A Chem profile, which includes sodium, potassium, chloride, carbon dioxide, Glucose, Urea, nitrogen, and creatinine is also obtained. Sodium, potassium, chloride, carbon dioxide are common electrolytes that can be out of range and contribute to problematic behaviors such as delirium. Urea nitrogen and creatinine are good indicators of dehydration. Thyroid screening with a T3 and T4 are also conducted. For the medication review, the NU evaluates potential inappropriate medications, poly-pharmacy and dosing using published reports. Within several days of the visit, the NU contacts the family by telephone to discuss laboratory results and in the case of positive results, coordinates a physician referral if the caregiver needs assistance. Caregivers are mailed two copies of laboratory results, one for their own records and the other to share with their family member's physician.

Following the initial OT and NU assessments, the OT continues working with the family caregiver at home. Over a series of visits and for each caregiver-identified problematic behavior, the OT provides: (1) education about the role of the environment, (2) skill-building in identifying antecedents to or triggers for the target behavior using a structured problem-solving approach, (3) specific strategies reflecting modifications to the physical and social environment to manage the behavior, and (4) stress reduction techniques. The OT provides a typed tailored action plan (one to three pages) which states the target behavior (eg, repetitive questioning), the target agreed upon treatment goals (eg, reduce frequency of occurrence of repetitive questioning in the morning and caregiver anger when behavior occurs), potential triggers that may contribute to the behavior (eg, feelings of despair and loss of control in person with dementia; unclear caregiver communication, difficulty way-finding in a cluttered environment; highly stressed caregiver), and directions for implementing customized strategies (eg, specific communication approaches, statements to avoid, use of tone and touch to provide reassurance, use of activities to engage person). As part of the treatment plan, caregivers may be provided up to $\$ 500$ in adaptive devices if necessary to address the problem behavior (eg, monitors, door locks, bathroom equipment). Caregivers build performance skills by practicing problem solving and strategy identification with the OT and then practicing strategies independently between scheduled sessions.

The maintenance phase occurs between study months 4 and 6 and involves three telephone contacts in which the OT reinforces strategy use, validates caregiver actions, and helps caregiver apply learned skills to newly emerging care problems.

\section{Intervention monitoring}

We use Lichstein and colleagues' fidelity model to monitor and quantitatively evaluate three treatment components: delivery (dose, intensity, mechanisms of delivery); receipt (caregiver acquisition of skills); and enactment (strategy 
Table I Overview of intervention protocol

\begin{tabular}{|c|c|c|c|c|}
\hline Session \# & Week \# & Type of contact & Interventionist & Overview of session content \\
\hline \multicolumn{5}{|c|}{ Active phase - Step I - Assessment of patient, caregiver and environment } \\
\hline I & 1 & Home visit & OT & - Rapport building \\
\hline & & & & $\begin{array}{l}\text { - Provide/review education material } \\
\text { Evaluate management techniques, } \\
\text { home environment, target behavior } \\
\text { and treatment goal }\end{array}$ \\
\hline 2 & 2 & Home visit & NU & - Rapport building \\
\hline & & & & $\begin{array}{l}\text { - Provide information about medical } \\
\text { contributors to behaviors } \\
\text { - Review patient medication } \\
\text { - Obtain urine and blood samples }\end{array}$ \\
\hline 3 & $3-4$ & Tele-contact & NU & $\begin{array}{l}\text { - Provide medical test results; physician } \\
\text { referral if necessary. }\end{array}$ \\
\hline \multicolumn{5}{|c|}{ Active phase - Step $2-$ Introduction of prevention and management techniques } \\
\hline 4 & 4 & Home visit & OT & $\begin{array}{l}\text { - Reinforce/facilitate physician referral } \\
\text { if necessary } \\
\text { - Use problem solving and brainstorming } \\
\text { (Ist target behavior) } \\
\text { - Identify potential strategies } \\
\text { - Introduce stress management technique }\end{array}$ \\
\hline 5 & $5-6$ & Home visit & OT & $\begin{array}{l}\text { - Introduce action plan and demonstrate, } \\
\text { role play each strategy }\end{array}$ \\
\hline 6 & $7-8$ & Home visit & OT & $\begin{array}{l}\text { - Review strategies and treatment goal } \\
\text { - Modify action plan if necessary } \\
\text { - Work on } 2 \text { nd target behavior as above } \\
\text { if Ist behavior resolved } \\
\text { - Introduce stress management technique }\end{array}$ \\
\hline 7 & $9-10$ & Tele-contact & OT & $\begin{array}{l}\text { - Review progress and use of strategies } \\
\text { - Reinforce use and practice of techniques }\end{array}$ \\
\hline 8 & $11-12$ & Home visit or tele-contact & OT & $\begin{array}{l}\text { - Review progress } \\
\text { - Reinforce use of strategies. } \\
\text { - Work on 3rd target behavior as above. }\end{array}$ \\
\hline 9 & $12-13$ & Home visit or tele-contact & OT & $\begin{array}{l}\text { - Review progress } \\
\text { - Target 4th behavior if appropriate } \\
\text { as above. }\end{array}$ \\
\hline 10 & $14-15$ & Home visit or tele-contact & OT & - Review progress/reinforce strategy use \\
\hline II & 16 & Home visit & OT & $\begin{array}{l}\text { - Review progress and strategy use } \\
\text { - Review problem solving steps. }\end{array}$ \\
\hline \multicolumn{5}{|c|}{ Maintenance - Step 3 - Closure and generalization to new problem areas } \\
\hline 12 & 18 & Tele-contact & OT & $\begin{array}{l}\text { - Review progress/reinforce strategy use } \\
\text { - Generalize strategies to new areas }\end{array}$ \\
\hline 13 & 21 & Tele-contact & OT & $\begin{array}{l}\text { - Review progress/reinforce use } \\
\text { - Validate caregivers skill }\end{array}$ \\
\hline 14 & 24 & Tele-contact & OT & - Review progress and obtain closure. \\
\hline
\end{tabular}

Abbreviations: OT, occupational therapist; NU, nurse.

use, caregiver report of problem resolution) (Lichstein et al 1994). The NU and OT interventionists document the target behavior, caregiver behavioral goals, specific strategies attempted and whether it contributed to resolution of the behavior and caregiver upset. Additionally, we use a combination of delivery and accuracy checklists to monitor randomly selected intervention sessions through audiotape and direct observation.

\section{Monitoring adverse events}

Although this study has minimal to no risk for participating families, as a Phase III randomized trial of efficacy, human subject safety oversight is provided by a Data and Safety Monitoring Board (DSMB). The DSMB is composed of three members representing an independent multidisciplinary group with biostatistical and behavioral research expertise represented. In monitoring safety of study participants (both 
caregiver and dementia patient), we distinguish between two event types: alerts, defined as a condition or event unrelated to study participation but which is encountered by a member of the research team (eg, medical emergency, unsafe home condition); and adverse, defined as events with some probable level of occurrence due to study participation (eg, injury from use of an environmental strategy recommended in the intervention) (Czaja et al 2006). The DSMB receives quarterly reports of recruitment progress (comparison of monthly expected to actual enrollment accrual) and alerts and their resolution. Adverse events are reported within 24 hours of occurrence to both the DSMB and the IRB.

\section{Measures}

Measures chosen for this study have known reliability and validity, demonstrated sensitivity to change, and relevance to the ACT intervention and caregiver research. We also sought to achieve a balance between psychometric quality and practical considerations such as administration time, respondent burden and special training needs of interviewers. We plan to examine the effect of the intervention on caregiver upset with targeted behaviors, and frequency of occurrence of these behaviors using an innovative targeted measurement approach.

\section{Targeted primary outcome measure of upset and behavioral frequency}

There is no single, universally accepted measure or methodology for operationalizing disruptive behaviors and caregiver upset (Tariot et al 1996). Research, especially in pharmacological interventions, has shown that the use of behavior inventories may mask important clinical effects of treatment (Mulsant et al 1997). This is due primarily to the fact that behavioral disturbance scales tend to be comprehensive, assessing diverse types of behaviors such as those that are psychiatric, affective or disruptive. Typically, scores are derived by summing across diverse behaviors to create a combined global rating. However, a person with dementia typically manifests only a few disruptive behaviors at any time point with most research showing an average of 3-4 behaviors occurring (Gitlin et al 2003; Sink et al 2006). Furthermore, an intervention designed to manage one behavioral domain (eg, psychiatric) may not effect other domains (eg, disruptive). Thus, to evaluate treatment outcomes, we have developed a target behavior approach that provides a measure that is both tailored to the family experience but which is standardized across study participants. A tailored measurement approach has been used extensively in medical and psychotherapeutic clinical trials (Koss et al 1983; Pilkonis et al 1984; Little and Rubin 1987; Battle et al 1996) and has been advocated as a reliable procedure for measuring change in the severity of behavioral occurrences in dementia patients (Mulsant et al 1997).

To derive a targeted measure, we first ask family caregivers to identify behaviors and the frequency of their occurrence ( 0 = did not occur in the week, $1=$ occurred 1-2 times in the week, 2 = occurred 3-6 times in the week, $3=$ occurred daily or more) using the ABID. Caregivers are then asked to rate their level of upset for each behavior that occurs $(0=$ no upset to $10=$ extreme upset with the midpoint labeled "fairly upset"). Subsequently, caregivers are asked to identify up to four of the most distressing behaviors that occur. A total target score is derived by summing across the upset responses for the one to four behaviors identified as most distressful (theoretical range of 0 to 40 , with higher scores indicating greater upset). Finally, caregivers are asked the extent to which they feel confident in handling targeted behaviors $(0=$ not confident to 4 = extremely confident). Thus, three family specific scores are obtained at baseline and then reassessed at the 4 and 6-month follow-ups: level of upset with up to four most distressful behaviors; frequency of occurrence of up to four distressing behaviors; level of confidence managing up to four distressful behaviors.

The target measurement approach has the advantage of pinpointing the behaviors that occur and from those, the ones that cause the most upset, avoiding reliance on an instrument which surveys a broad range of problems, most of which may not be problematic to any one caregiver, or an instrument that too narrowly focuses on a limited domain of behaviors. As such, this measurement approach provides a systematic yet customized strategy with high clinical relevance. A target outcome approach also is effective in dementia caregiving research because the universe of possible disruptive behaviors, although theoretically unlimited, is in practice finite and definable. It is possible that other behaviors become more problematic at subsequent testing occasions. We will therefore also examine pre-post change in frequency of behavior occurrence and caregiver upset using the total ABID scale score to ensure that information about emergent problem behaviors and associated upset is captured.

\section{Conclusion}

BPSD are common and these behaviors have a profound impact on families. Pharmacological treatments are useful but for a limited type of behaviors. Current research suggests that BPSD reflect the interplay of pathology and 
environmental factors and that intervention needs to be developed from that perspective. Project ACT is a relatively brief, focused intervention that is designed to help families identify potential modifiable triggers of targeted behaviors and modification strategies to minimize their occurrence. Different treatment modalities including medical testing, education, problem solving, customized action plans, demonstration and role play using environmental modifications are introduced to provide a comprehensive approach to helping families learn new skills to cope with this significant domain of concern.

The study of whether a targeted, multi-disciplinary treatment approach can prevent, reduce or manage behavioral occurrences in persons with dementia and minimize family caregiver distress has major public health and social policy import. A positive result from this trial would provide an evidence-based intervention that could easily be translated into clinical practices of primary and home care providers. It would elevate clinical practice in the management of dementia patients and offer clinicians a positive, low cost approach to improving life quality of families struggling with this devastating disease. Alternately, failure to reject the null hypothesis may suggest that treatment effects occur but outcomes measures are not sensitive to behavioral change, the benefits are in areas not measured, or that behaviors and caregiver distress can not be minimized by modifying the physical and social environment such that efforts would need to be directed elsewhere to have an impact on behavior management.

\section{References}

Ballard C, Lowery K, Powell L, et al. 2000. Impact of behavioral and psychological symptoms of dementia on caregivers. Int Psychogeriatr, 12:93-105.

Baron RM, Kenny DA. 1986. The moderator-mediator variable distinction in social psychological research: Conceptual, strategic, and statistical considerations. J Pers Soc Psychol, 51:1173-82.

Battle CC, Imber SD, Hoen-Saric R, et al. 1966. Targets complaints as criteria of improvement. Am J Psychother, 20:184-92.

Baumgarten M, Becker R, Gauthier S. 1990. Validity and reliability of the Dementia Behavior Rating Scale. J Am Geriatr Soc, 38:221-6.

Boucher LA. 1999. Disruptive behaviors in individuals with Alzheimer's disease: a behavioral approach. Am J Alzheimer Dis, 14:351-6.

Burton LC, Rovner BW, German PS, et al. 1995. Neuroleptic use and behavioral disturbance in nursing homes: A one year study. Int Psychogeriatr, 7:535-45.

Calkins MP. 1989. Design for dementia. Owings Mills, MD: National Health.

Class CA, Schneider L, Farlow MR. 1997. Optimal management of behavioral disorders associated with dementia. Drugs Aging, 10:95-106.

Cohen-Mansfield J. 2001. Nonpharmacologic interventions for inappropriate behaviors in dementia: a review, summary, and critique. Am J Geriatr Psychiatry, 9:361-81.

Cohen-Mansfield J, Marx MS, Gauthier S. 1989. A description of agitation in a nursing home. J Gerontol, 44:M77-84.
Colenda CC III. 1995. Agitation: A conceptual overview. In: Lawlor BA ed. Behavioral complications in Alzheimer's disease. Washington, DC: American Psychiatric Press, Inc. p 3-17.

Corcoran MA, Gitlin LN. 1991. Environmental influences on behavior of the elderly with dementia: Principles for intervention in the home. Phys Occup Ther Geriatr, 9:5-21.

Czaja SJ, Schulz R, Belle SH, et al. 2006. Data safety monitoring in social behavioral intervention trails: the REACH II experience. Clinical Trials, 3:107-18.

Finkel SI, Burns A eds. 2000. Behavioral and psychological symptoms of dementia (BPSD): a clinical and research update. Int Psychogeriatr, 12:9-18.

Folstein MF, Bylsma FW. 1999. Noncognitive symptoms of Alzheimer's disease. In: Terry RD, Katzman R, Bisk KL, et al eds. Alzheimer disease. Philadelphia: Lippincott Williams and Wilkins. p 25-37.

Gitlin LN, Liebman J, Winter L. 2003. Are environmental interventions effective in the management of Alzheimer's disease and related disorders? A synthesis of the evidence. Alzheimer Care Quarterly, 4:85-107.

Gitlin LN, Winter L, Corcoran M, et al. 2003. Effects of the home environmental skill-building program on the caregiver-care recipient dyad: six-month outcomes from the Philadelphia REACH Initiative. Gerontologist, 43:532-46.

Gitlin LN, Corcoran MA, Winter L, et al. 2001. A randomized, controlled trial of a home environmental intervention: effect on efficacy and upset in caregivers and on daily function of persons with dementia. Gerontologist, 4:15-30.

Haley WE, Bailey S. 1999. Research on family caregiving in Alzheimer's disease: Implications for practice and policy. In: Vellas B, Fitten JL eds. Research and practice in Alzheimer's disease. Paris: Serdi Publisher. Vol 1:p 321-32.

Hall GR, Buckwalter KC. 1987. Progressively lowered stress threshold: a conceptual model for care of adults with Alzheimer's disease. Arch Psychiatr Nurs, 1:399-406.

Koss MP, Graham JR, Kirkhart K, et al. 1983. Outcome of eclectic psychotherapy in private psychological practice. Am J Psychother, 3:400-10.

Lawton MP. 1982. Competence, environmental press, and the adaptation of older people. In: Lawton MP, Windley PG, Byerts TO eds. Aging and the environment: theoretical approaches. New York: Springer Publishing Company. p 33-59.

Lawton MP, Nahemow LE. 1973. Ecology and the aging process. In: Eisdorfer C, Lawton MP eds. The psychology of adult development and aging. Washington, DC: American Psychological Association. p 619-74.

Lichstein JL, Riedel BW, Griev R. 1994. Fair tests of clinical trials: a treatment implementation model. Adv Behav Res Ther, 16:1-29.

Little RJA, Rubin DB. 1987. Statistical analysis with missing data. New York: John Wiley and Sons.

Logsdon RG, Teri L, Weiner MF, et al. 1999. Assessment of agitation in Alzheimer's disease: the agitated behavior in dementia scale. $J \mathrm{Am}$ Geriatr Soc, 47:1354-8.

Lyketsos CG, Breitner JCS, Rabins PV. 2001. An evidence-based proposal for the classification of neuropsychiatric disturbance in Alzheimer's disease. Int J Geriatr Psychiatry, 16:1037-42.

Lyketsos CG, Sheppard JME, Steinberg M, et al. 2001. Neuropsychiatric disturbance in Alzheimer's disease clusters into three groups: the Cache County study. Int J Geriatr Psychiatry, 16:1043-53.

McCarty HJ, Roth DL, Goode KT, et al. 2000. Longitudinal course of behavioral problems during Alzheimer's disease: Linear versus curvilinear patterns of decline. J Gerontol A Biol Sci Med Sci, 55A: M200-6.

Mittelman MS. 2000. Nonpharmacologic management and treatment: effect of support and counseling on caregivers of patients with Alzheimer's disease. Int Psychogeriatr, 12:341-6.

Moniz-Cook E, Vernooij-Dassen M. 2006. Timely psychosocial intervention in dementia: a primary care perspective. Dementia, 5:307-15. 
Mulsant BH, Mazumdar S, Pollack BG, et al. 1997. Methodological issues in characterizing treatment response in demented patients with behavioral disturbances. Int J Geriatr Psychiatry, 12:537-47.

Pilkonis PA, Imber SD, Lewis P, et al. 1984. A comparative outcome study of individual, group, and conjoint psychotherapy. Arch Gen Psychiatry, 41:431-7.

Reisberg B, Borenstein J, Salob SP, et al. 1987. Behavioral symptoms in Alzheimer's disease: phenomenology and treatment. J Clin Psychiatry, 48:9-15.

Schneider LS, Pollock VE, Lyness SA. 1990. A meta-analysis of controlled trials of neuroleptic treatment in dementia. J Am Geriatr Soc, 38:553-63.

Schulz R, Gallagher-Thompson D, Haley W, et al. 2000. Understanding the intervention process. A theoretical/conceptual framework for intervention approaches to caregiving. In: Schulz R ed. Handbook on Dementia caregiving. Springer Publ. pp 33-40.
Sink KM, Covinsky KE, Barnes DE, et al. 2006. Caregiver characteristics are associated with neuropsychiatric symptoms of dementia. $J \mathrm{Am}$ Geriatr Soc, 54:796-803.

Swanwick GRJ. 1995. In: Lawlor BA ed. Nonpharmacological treatment of behavioral symptoms. Washington, DC: American Psychiatric Press, Inc. p 183-207.

Swearer JM, Drachman DA, O’Donnell BF, et al. 1988. Troublesome and disruptive behaviors in dementia: Relationships to diagnosis and disease severity. J Am Geriatr Soc, 36:784-9.

Tariot PN, Porsteinsson A, Teri L, et al. 1996. Measurement of behavioral disturbance in chronic care populations. J Mental Health Aging, 2:213-29. 
